International Journal of Wireless \& Mobile Networks (IJWMN) Vol. 3, No. 3, June 2011

\title{
ROUTING TECHNIQUES IN COGNITIVE RADIO NETWORKS: A SURVEY
}

\author{
Amjad Ali ${ }^{1}$ Muddesar Iqbal ${ }^{2}$ Adeel Baig ${ }^{1}$ Xingheng Wang ${ }^{3}$ \\ ${ }^{1}$ School of Electrical Engineering and Computer Sciences National University of \\ Science and Technology, Pakistan \\ Email: \{amjad.ali, adeel.baig\} @seecs.edu.pk \\ ${ }^{2}$ Faculty of Computer Science \& Information Technology, University of Gujrat, \\ Pakistan \\ ${ }^{3}$ College of Engineering, Swansea University, Swansea, UK \\ Email: xingheng.wangeswansea.ac.uk
}

\begin{abstract}
Cognitive Radio Networks (CRNs) are being studied intensively. The major motivation for this is the heavily underutilized frequency spectrum. CRN has the capability to utilize the unutilized frequency spectrum. Routing in CRN is a challenging task due the diversity in the available channels and data rates. In this paper, we present a survey of the state-of-the-art routing techniques in CRNs. We first outline the design challenges for routing protocols in CRNs followed by a comprehensive survey of different routing techniques. Furthermore we classified these routing protocols into spectrum aware-based, multipathbased, local coordination-based, reactive source-based and tree-based routing techniques depending on the protocol operation.
\end{abstract}

\section{KEYWORDS}

CRNs, DSA, RF spectrum, Routing

\section{INTRODUCTION}

Cognitive Radio Networks (CRNs) can operate in the licensed frequency band to improve its utilization with the coexistence of the Primary Users (PRs) or licensed users. PRs have the main rights over the licensed band in which they are operating.

Radio frequency (RF) is an important resource that people uses all around the world for many services i.e. safety, communication, employment, and entertainment [1].The dedicated frequency band is allocated to the paid user that uses this frequency for specific service. Thus the RF band allocated can be vastly underutilized. Recent studies show that only $5 \%$ of the spectrum from $30 \mathrm{MHz}$ to $30 \mathrm{GHz}$ is used in the US [2]. The Federal Communications Commission (FCC) of United States of America found that spectrum usage is a more significant problem than the actual physical availability of RF spectrum [3]. The spectrum availability problem arises due to the currently deployed static spectrum allocation policy that limits the usage of the licensed RF band only to the licensed user or primary user. These findings need more efficient methods for utilization of the RF resources and the Cognitive Radio (CR) technology is envisioned as new mechanism for flexible usage of the RF spectrum. This technology enables the secondary users or unlicensed users to operate in the licensed band with the coexistence of the licensed users or primary users. Secondary users have the ability to identify and utilize the available channels in the RF spectrum. The ability of a secondary user to change its frequency of operation is commonly referred as dynamic spectrum access (DSA). Thus we can say that cognitive network is a network that can observe current network 
conditions, and then act on those conditions. The network can learn from these actions and use them to make future decisions [4]. Ryan W. Thomas and Daniel H, define the CRNs in the context of machine learning as "cognitive network improves its performance through experience gained over a period of time without complete information about the environment in which it operates" [5]. Thus a secondary user can change its transmitter parameters based on its learning from the environment, based on these changes the secondary user can efficiently utilize the frequency band and avoid from the interference on the primary user.

The main feature of CR technology that how it operates in the licensed band with the coexistence of the PR users is that it identifies the spectrum holes in the RF spectrum called white spaces. These white spaces are the wastage in the RF spectrum and would be used by secondary user for its communication.

Figure 1shows the concept of spectrum holes.

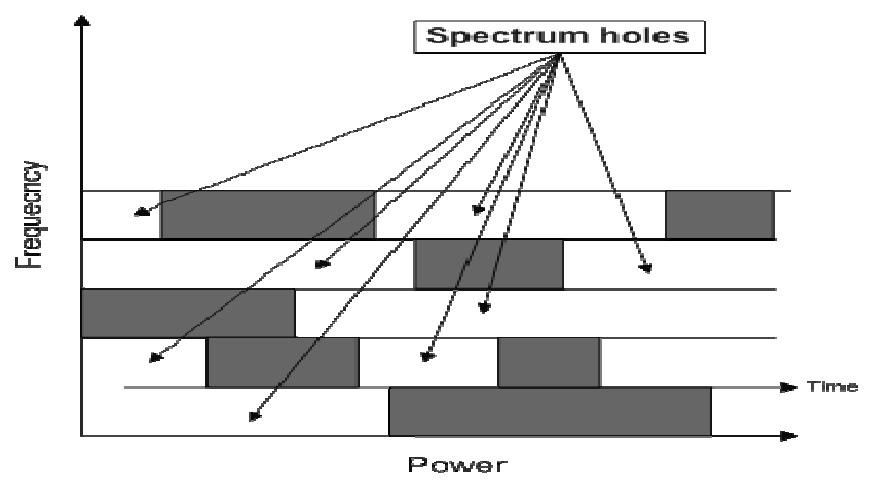

Figure 1: Spectrum holes concept

The rest of this paper is organized as follows. In Section 2, we discuss the CRNs architecture. In section 3 , we discuss routing differences and challenges. A comprehensive survey of routing techniques in CRNs is presented in Section 4.

\section{ArChitecture For CRNs}

A clear description of the CRNs architecture is important for the understanding of their working and designing novel protocol for communications. Figure 2 describes the architecture of the CRNs. 


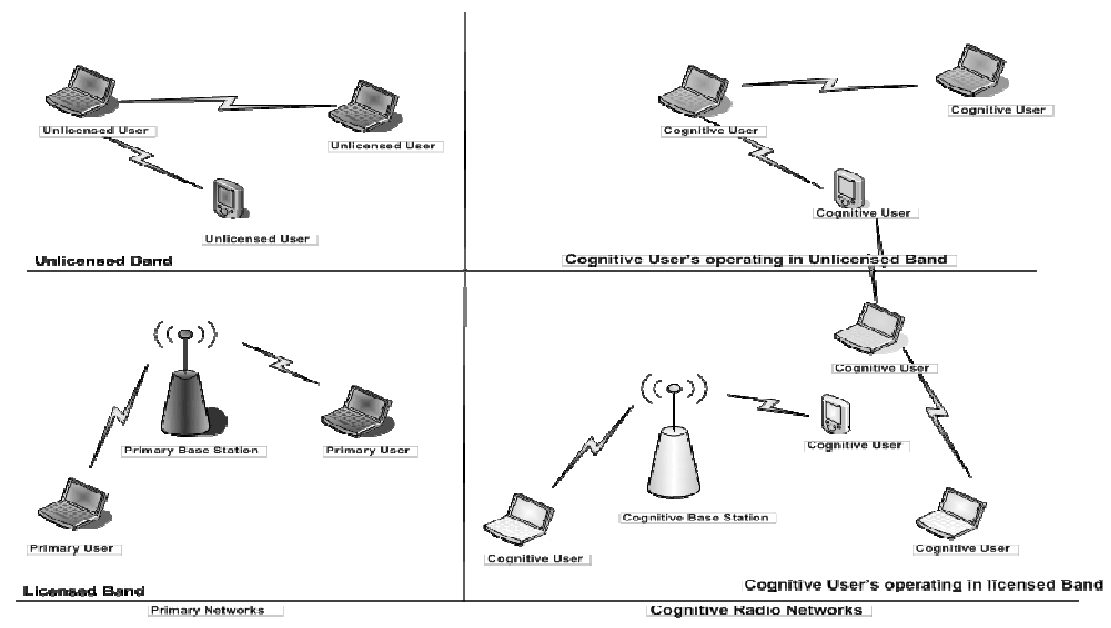

Figure 2: Cognitive network architecture.

The CRNs can be classified into two main categories:

a. Infrastructural CRN

b. Infrastructure less CRN

Infrastructural CRN: In this type of CRN the communication is done with the help of a fixed infrastructure component called CRN base station.

Infrastructure less CRN: In this type of CRN the communication is done without the support of fixed infrastructure or base station. This type is similar to ad hoc multi-hop network.

The elements of the primary and the cognitive network are defined as follows:

Primary Network: A network that owns a separate RF spectrum band for its services (e.g. common cellular and TV broadcast networks). The components of the primary network are as follows:

Primary User: Primary user or licensed user has main rights to operate in its RF spectrum band. The primary user cannot bear any interference on its RF band due to any secondary user.

Primary Base Station: It is a fixed infrastructure component that controls the access of its licensed users. This component does not have any ability to share RF spectrum band with CR users.

Cognitive Network: Cognitive network does not have any RF spectrum band for their communication. That's why these networks are called unlicensed networks.

The components of cognitive networks are as follows:

Cognitive User: Cognitive user does not have its own frequency band; therefore these users are called unlicensed users or secondary users. These users only share the licensed band in an opportunistic manner. Secondary user requires extra functionality such as spectrum sensing to operate in licensed band.

Cognitive Base Station: Cognitive base station is a fixed infrastructure component similar to primary base station with cognitive capabilities. The secondary users access other networks through it. It provides single hope connection to secondary users. 
International Journal of Wireless \& Mobile Networks (IJWMN) Vol. 3, No. 3, June 2011

\section{Routing DifFerences AND ChALLENGES IN CRNS}

Conventional infrastructure networks and mobile ad hoc networks have been studied since last decade and many routing protocols, e.g. proactive, reactive, hierarchical and multicast are available for such networks [6].

Now it's time to investigate the novel routing protocols for CRNs, as routing is a challenging task in such networks, particularly in multi hop CRNs due to the diversity in channel availability and data rates. Multi-interface enabled CR user can avail multiple available channels simultaneously, thus increase overall network performance and reduce the interference on the primary users. Due to this vital feature of CRNs conventional routing metrics such as hope count, congestion, etc, are not sufficient for routing decision in CRNs [6].

The primary differences and challenges between routing of CRNs and routing of conventional wireless networks can be summarized as follows:

\subsection{Link Availability:}

DSA senses the RF band and fetches the available opportunities. Thus these networks use the licensed band in opportunistic manner for communication. These communication opportunities are available when primary users have fewer usage of their frequency band or they are not using it at all, therefore availability of channel is time and geographic based. Due to this random availability of channel the CRNs topology is random even all communicating nodes are static [7].

\subsection{Deafness Problem:}

Due to the diversity in channel or link availability, links may be available only a fraction of time in CRNs. This random availability may cause the deafness problem, which is switching among available channel set to maintain the route or avoid the interference on primary users. This causes extra delay in CRNs communications [8].

\subsection{Unidirectional Links:}

In CRNs, unidirectional links are more likely to be happened. In CRNs links are only available for a fraction of time instead of minutes or hours. This cause's unidirectional links as there is no guarantee that a channel used for sending station will be available till the receiving station uses the same channel for transmission.

\subsection{Heterogeneous Wireless Networks:}

CRNs are usually come into existence by multiple and heterogeneous wireless networks. Therefore intersystem handover is critical and required for routing. Along with this channels or links are available for an extremely short duration; therefore successful networking lies in cooperative relaying among such heterogeneous wireless networks [9].

\subsubsection{Security issues:}

Enabling of CRNs at the price of losing security is a debatable issue. Short duration of available channel is not a sufficient for CR user or node to get secure certificate.

\section{Routing Protocols For CRNs}

In this section, we survey the state-of-the-art routing protocols for CRNs. We classified these routing protocols into spectrum aware-based, multipath-based, local coordination-based, reactive source-based and tree-based routing techniques depending on the protocol operation as shown in figure3. Furthermore we presented protocol summary in the form of tables. 


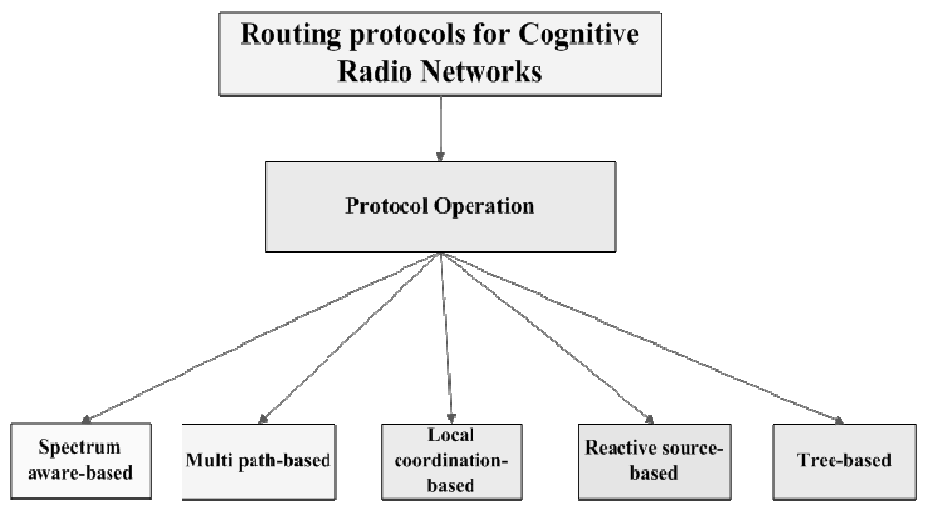

Figure 3: Routing protocol for CRNs

\subsection{Dynamic Spectrum-aware Routing}

Dynamic spectrum-aware routing protocols enable CR technologies to effectively utilize unallocated wireless spectrum. In such routing protocols route discovery is incorporated with spectrum sensing. The main goal of such protocols is to establish and maintain route across region of different available spectrum. In the rest of the sub section we summarize these protocols and highlight their advantages and their routing techniques. Protocol summery in the form of table is presented at the end of each protocol.

\subsubsection{SPEctrum-Aware Routing (SPEAR)}

A routing protocol that supports high-throughput packet transmission in the presence of spectrum heterogeneity is being investigated in [10]. It achieves persistent end-to-end performance by integrating flow-based approaches with link-based approaches. It assigns different channels to links on the same flow for minimizing interference and integrates spectrum discovery with route discovery for optimal usage of available channels. For this, each node maintains a list of unoccupied locally available channels. These channels are neither occupied by primary user nor reserved by nearby neighbors. In SPEAR route discovery is done by broadcasting a Route Request (RREQ) message on common control channel and being identified by sender and receiver IP addresses. As an intermediate node receives this message, it checks if it has a common channel with the previous node then it appends its own id and available channel set with the received message and then broadcasts it. The destination node selects the best path on the basis of maximum throughput, minimizes end-to-end latency (minimum hop count) and link quality. During the transmission node periodically broadcasts channel reservation message with each message containing timeout and time-to-live field. At the end of communication nodes along the path are notified to stop sending reservation messages.

\subsubsection{Advantages and Overhead}

SPEAR distributed on demand routing protocol that utilizes the spectrum heterogeneity. It has low computational and communication complexity. It almost achieves end-to-end throughput near optimal and adding additional nodes does not degrade its performance. Timeout field introduced in route set up simplifies management and provides robustness against link failure. Similarly time-to-live field minimizes broadcast overhead and contention. This protocol can be used with the narrow band control channel. On demand route discovery measured in term of route set up and route tear down delay and periodic channel reservation messages involves additional overhead. 


\subsubsection{Spectrum Aware MEsh Routing (SAMER)}

A routing protocol for mesh CRNs proposed in [11] handles the diversity in channel availability and balance between long-term route stability and short-term route. SAMER uses the available white spaces by transmitting the data over the route with higher spectrum availability. Thus spectrum availability is used for computing routing metric for long-term routes. It achieves the balance between long- and short-term routes by constructing a runtime forwarding route mesh. This mesh is periodically updated and provides a set of candidate routes to the destination. Thus packets are routed towards the destination across this mesh. The routing decisions are taken with the collaboration of PHY and MAC layer. SAMER builds dynamic candidate, candidate forwarding mesh and opportunistically forwarding.

\subsubsection{Dynamic Candidate Mesh:}

In constructing dynamic candidate mesh a cost to destination is computed by every node in the network. The cost actually shows the spectrum availability of the highest spectrum route. This route contains fewer hops than a specified threshold. Thus each node constructs a set of forwarding nodes to destination.

Opportunistic Forwarding: SAMER uses those links for forwarding that contains the highest spectrum availability. It used PSA metric which is defined in term of throughput between a pair of nodes for computing spectrum availability.

\subsubsection{Building a candidate forwarding mesh:}

Forwarding mesh only contains long-term paths. These paths are the shortest in term of hope count. Thus for forwarding packet to destination D a node i computes a cost for all of its neighboring nodes and it adds only those nodes in its forwarding set that contain minimum cost. Therefore a cost to destination is prior computed by each network node.

\subsubsection{Advantages:}

SAMER achieves high end-to-end throughput by using long-term stability and short-term opportunistic utilization of spectrum. Changes in spectrum availability are adopted dynamically by its forwarding mesh.

Table 1: Summary of SPEAR\& SAMER Routing Protocols.

\begin{tabular}{|l|l|l|l|l|}
\hline & \multicolumn{2}{|l|}{ SPEAR } & & \multicolumn{3}{|c|}{ SAMER } \\
\hline $\begin{array}{l}\text { Objective } \\
\text { and features }\end{array}$ & & Technique & & Technique \\
\hline $\begin{array}{l}\text { End-to-end } \\
\text { throughput }\end{array}$ & Yes & $\begin{array}{l}\text { Integration of } \\
\text { flow-based and } \\
\text { link-based } \\
\text { approaches }\end{array}$ & Yes & $\begin{array}{l}\text { Path with high spectrum availability, long- } \\
\text { term stability and short-term opportunistic } \\
\text { utilization of spectrum }\end{array}$ \\
\hline $\begin{array}{l}\text { Route } \\
\text { discovery }\end{array}$ & $\begin{array}{l}\text { Control } \\
\text { channel, } \\
\text { Broadcasting } \\
\text { RREQ message }\end{array}$ & & Link state packets \\
\hline $\begin{array}{l}\text { Routing } \\
\text { decisions }\end{array}$ & $\begin{array}{l}\text { With the } \\
\text { Collaboration } \\
\text { of PHY and }\end{array}$ & & $\begin{array}{l}\text { With the Collaboration of PHY and MAC } \\
\text { layers }\end{array}$ \\
\hline
\end{tabular}


International Journal of Wireless \& Mobile Networks (IJWMN) Vol. 3, No. 3, June 2011

\begin{tabular}{|c|c|c|c|c|}
\hline & & MAC layers & & \\
\hline Route nature & & On demand & & Periodical \\
\hline $\begin{array}{l}\text { Mobility } \\
\text { handling }\end{array}$ & Yes & $\begin{array}{l}\text { Timeout field } \\
\text { in periodic } \\
\text { channel } \\
\text { reservations } \\
\text { messages }\end{array}$ & No & \\
\hline $\begin{array}{l}\text { Communicati } \\
\text { on overhead }\end{array}$ & Yes & $\begin{array}{l}\text { Route setup, } \\
\text { route tear down }\end{array}$ & Yes & $\begin{array}{l}\text { Each node computes its cost to } \\
\text { destination, }\end{array}$ \\
\hline $\begin{array}{l}\text { Computation } \\
\text { al } \\
\text { Complexity }\end{array}$ & Low & & Low & \\
\hline $\begin{array}{l}\text { Route } \\
\text { discovery } \\
\text { packet size }\end{array}$ & $\begin{array}{l}\text { Variabl } \\
\text { e }\end{array}$ & $\begin{array}{l}\text { Each node } \\
\text { appends its } \\
\text { identifier and } \\
\text { available } \\
\text { channel set }\end{array}$ & Fixed & Hop-to-hop calculation \\
\hline $\begin{array}{l}\text { Best path } \\
\text { selection }\end{array}$ & Yes & $\begin{array}{l}\text { Maximum } \\
\text { throughput, } \\
\text { minimum hop } \\
\text { count and link } \\
\text { quality }\end{array}$ & Yes & $\begin{array}{l}\text { Minimum hop count and spectrum } \\
\text { availability }\end{array}$ \\
\hline
\end{tabular}

\subsubsection{Spectrum-aware On-Demand routing protocol (SORP)}

SORP is an on demand routing protocol that is neither based on centralized spectrum allocation nor multi-channel. The nature of this protocol is due to lack of shared information. The routing technique proposed by Cheng et.al in [12] is to select best suitable RF bands for each node along the route. The RF band selection is based on minimum cumulative delay. The switching and back-off delay caused by both the path itself and the intersecting flow are the judging parameters for calculating cumulative delay of the path. They proposed a spectrum aware on demand framework for routing and multi-flow multi-frequency scheduling for RF band selection. They slightly modified Ad hoc on demand distance vector routing (AODV) [20] to incorporate the inconsistency of spectrum opportunity. They made some assumptions for their routing technique, as follow:

To form a common control channel each node contains a traditional wireless interface in addition to the CR transceiver. Each node is able to provide spectrum sensing information to routing protocol through cross layer design.

For route discovery SORP inherits the basic procedures of AODV with modified Route Request (RREQ).In SORP Spectrum Opportunity (SOP) information is piggybacked by RREQ messages. SOP information is piggybacked only when the node finds intersection between the RREQ and its own. Thus destination node receives the SOP distribution of all the nodes along 
the path and it assigns RF band to its CR transceiver accordingly. This RF band information is sent back to the source node as well intermediate nodes through Route Reply (RREP) message. All the nodes along the path assign the RF band according to the received RREP.

\subsubsection{Advantages and Overhead:}

This routing technique overcomes the inconsistency of SOPs. It selects best path on the basis of the total delay along the path. It selects the RF band with joint interaction of routing and scheduling. Therefore path cumulative based RF band selection introduces both switching and back off delay.

\subsubsection{Multi-hop Single-transceiver Cognitive Radio Networks Routing Protocol (MSCRP):}

MSCRP proposed in [13] doesn't base on control channel. Therefore, routing protocol messages are being exchanged without common control channel. MSCRP is an on demand protocol based on ad hoc on demand distance vector (AODV).

Ma et.al modifies AODV to handle the available channel set problem that each node in the network doesn't know the available channel set of other nodes in the network. In [13] they first time introduced the new problem called "deafness", that is due to channel switching of the nodes. To avoid the deafness problem, they proposed that two consecutive nodes in a flow cannot be in the switching state simultaneously. Communicating with a switching node is complicated, therefore MSCRP switching node uses LEAVE/JOIN messages to inform its neighbors about its working channel. MSCRP assumes that CR transceiver can tune in a wide range of RF spectrum but it only operates on limited and smaller range of RF and CR transceiver can only operate on single channel at any time. MSCRP is a cross layer protocol so it identifies six system functions that implement the core functionality of spectrum aware routing. These functions are as follow:

The physical layer includes three of them that are spectrum sensing, detecting active primary user and estimating the quality of available channels.

The network layer includes two of them that are routing and scheduling in the multi-flow and multi-channel environment.

Link layer has last one that is IEEE 802.11DCF is used as the MAC protocol.

\subsubsection{Route Discovery Mechanism in MSCRP:}

RREQ message is broadcast on all the channels for route discovery. The channels availability information piggybacked by RREQ a message is forwarded in broadcast process. All intermediate nodes append their state and available channel set to RREQ message. As nodes may stay on different channels, therefore broadcast manner is totally different from original broadcast used in AODV. The reverse path to the source node is established as RREQ is forwarded. Destination node receives the channel information and number of nodes on each channel at the end and assigns channel for this flow. It encapsulates the assigned channel information in RREP message.

\subsubsection{Advantages and Overhead:}

It first time introduces the deafness problem and introduces a novel approach to deal it. This approach is well fit in multi-hop and single transceiver CRNs. Dealing with single transceiver is cost-effective as compared to multi-transceiver. The deafness introduces some extra delay to RREQ messages due to its channel switching. MSCRP also introduces extra overhead of broadcasting RREQ message on all available channels rather on single channel and this overhead will becomes insufferable in case many available channels on each network node. 
Table 2: Summary of SORP \& MSCRP Routing Protocols.

\begin{tabular}{|c|c|c|c|c|}
\hline & \multicolumn{2}{|l|}{ SORP } & \multicolumn{2}{|l|}{ MSCRP } \\
\hline $\begin{array}{l}\text { Objective and } \\
\text { features }\end{array}$ & & Technique/ explanation & & $\begin{array}{l}\text { Technique/ } \\
\text { explanation }\end{array}$ \\
\hline $\begin{array}{l}\text { End-to end } \\
\text { throughput }\end{array}$ & Yes & $\begin{array}{l}\text { spectrum aware } \\
\text { on demand routing and } \\
\text { multi-flow multi-frequency } \\
\text { scheduling }\end{array}$ & Yes & $\begin{array}{l}\text { Spectrum aware routing } \\
\text { and leave/join messages }\end{array}$ \\
\hline Route discovery & & Broadcast RREQ messages & & $\begin{array}{l}\text { RREQ message on all } \\
\text { available channels rather } \\
\text { on single channel }\end{array}$ \\
\hline $\begin{array}{l}\text { Routing } \\
\text { decisions }\end{array}$ & & $\begin{array}{l}\text { With the collaboration of } \\
\text { MAC and network layers }\end{array}$ & & $\begin{array}{l}\text { With the collaboration } \\
\text { of MAC, physical and } \\
\text { network layers }\end{array}$ \\
\hline Route nature & & On demand & & On demand \\
\hline $\begin{array}{l}\text { Link failure } \\
\text { handling }\end{array}$ & No & & Yes & Leave/join messages \\
\hline $\begin{array}{l}\text { Communication } \\
\text { overhead }\end{array}$ & & $\begin{array}{l}\text { Path cumulative based RF } \\
\text { band selection introduces } \\
\text { both switching and back off } \\
\text { delay }\end{array}$ & & $\begin{array}{l}\text { Deafness introduces } \\
\text { extra delay to RREQ } \\
\text { due to its channel } \\
\text { switching and } \\
\text { broadcasting RREQ } \\
\text { messages on all } \\
\text { available channels } \\
\text { introduce extra overhead }\end{array}$ \\
\hline $\begin{array}{l}\text { Route discovery } \\
\text { packet size }\end{array}$ & Variable & $\begin{array}{l}\text { Each node appends SOP } \\
\text { list }\end{array}$ & Variable & $\begin{array}{l}\text { All intermediate nodes } \\
\text { append their state } \\
\text { information and } \\
\text { available channel set }\end{array}$ \\
\hline $\begin{array}{l}\text { Best path } \\
\text { selection }\end{array}$ & Yes & $\begin{array}{l}\text { Path delay and node delay } \\
\text { (switching and back off } \\
\text { delay) }\end{array}$ & Yes & $\begin{array}{l}\text { Number of flows on } \\
\text { each channel }\end{array}$ \\
\hline Control channel & Yes & $\begin{array}{l}\text { Exchanging the routing } \\
\text { protocol messages }\end{array}$ & No & $\begin{array}{l}\text { Data channel for routing } \\
\text { protocol messages }\end{array}$ \\
\hline
\end{tabular}

\subsection{Reactive Source-Based Routing}

In reactive source based routing technique source specifies how the data travels across the network. Path to destination node is computed by the source node. In the rest of the sub section we summarize a reactive source-based routing protocol and highlight its routing technique and its advantages. 


\subsubsection{Routing in Opportunistic Cognitive Radio Networks}

Reactive source-based routing protocol for CRNs is proposed by Khalife et.al [14] and it uses a novel routing metric that is based on a probabilistic definition of the available capacity over a channel. This routing metric determines the most probable path (MPP) to satisfy a given bandwidth demand although it doesn't guarantee to satisfy the demand. So in this case an augmentation phase is used in which bottleneck links are augmented with additional channels so the resulting path meets the bandwidth demand with a given probability.

The available capacity is measured as the probability distribution of the PR to CR user interference at any node over a channel.

When an application requests a route of capacity demand the source will initiate it and control channel is used for node coordination. Based on the demand all links probabilities are calculated. Once all link weights are calculated, the source runs Dijkstra-like algorithm to find a route to the destination. The obtained path is called MPP as it has the highest probability of satisfying the demand and stability to destination. The Dijkstra-like algorithm stops computing when it reaches to the one of the following two states.

1. On each link of MPP, the total capacity will be greater than the demand.

2. After augmentation if the total estimated capacity on all the channels of two nodes will not fulfill the demand. In this case no path is suitable to the destination thus it is declared unreachable.

\subsubsection{Advantages:}

This routing protocol deals with the simultaneous transmissions over multiple channels as well Primary Radio-to-Cognitive Radio interference.

Table3: Summary of Reactive Source based Routing Protocol.

\begin{tabular}{|l|l|l|}
\hline Objective and features & & Technique /explanation \\
\hline End-to-end throughput & Yes & $\begin{array}{l}\text { Selecting MPP path that fulfils the application capacity } \\
\text { demand }\end{array}$ \\
\hline Route discovery & & OSPF, Dijkstra-like algorithm to compute the route \\
\hline Routing decisions & & Does not base on cross layer \\
\hline Route nature & & On demand \\
\hline Data structure & & Graph \\
\hline Link weight assignment & Yes & Based on available capacity of link \\
\hline link failure handling & No & \\
\hline Route discovery packet size & Fixed & Hop-to-hop communication \\
\hline Best path selection & Yes & $\begin{array}{l}\text { A routing metric based on the probabilistic definition of } \\
\text { available capacity over channel }\end{array}$ \\
\hline Control channel & Yes & \multicolumn{2}{|l}{} \\
\hline
\end{tabular}

\subsection{Local Coordination-Based Routing}

The local coordination is a sort of enhancement scheme that is applied on intersecting nodes on a path. The local coordination is started when nodes evaluate the workload of both 
accommodating the flow and redirecting it. Nodes choose the flow accommodation or flow redirection based on the evaluation results and neighborhood interaction.

In the rest of the sub section we summarize a local coordination-based routing protocol and highlight its routing technique and its advantages.

\subsubsection{Local Coordination Based Routing and Spectrum Assignment in Multi-hop Cognitive Radio Networks:}

An on demand routing and spectrum assignment protocol to exchange the local spectrum information and interact with multi-frequency scheduling in each node is proposed by Yang et.al [15]. AODV is modified to form a mechanism on common control channel for exchanging spectrum opportunity (SOP) among the nodes to overcome the inconsistency of SOP. It also identifies traversing flows at every node and calculates RF band used by any node and this is used for multi-flow multi-frequency scheduling. Path delay and node delay show the switching and back off delays along the path and used to calculate the cumulative delay of the path. A local coordination scheme is used for load balancing on intersecting nodes for multi-frequency traffic. Each network node is equipped with traditional wireless interface in addition to CR transceiver to ensure the successful delivery of routing messages at each node despite of the inconsistency of the frequency bands as well every node provides the SOP information to its network layer. The local coordination is applied on every network node of multi-hop CRNs.

\subsubsection{Advantages:}

The proposed routing protocol provides good adaptability for spectrum diversity and end-to-end delay. This scheme outperforms the traditional bare routing.

Table 4: Summary of LCB Routing Protocol.

\begin{tabular}{|c|c|c|}
\hline Objective and features & & Technique /explanation \\
\hline End-to-end delay & Less & Adaptive relay is cooperating with routing protocol \\
\hline End-to-end performance & & $\begin{array}{l}\text { Redirecting flow to other neighboring nodes and } \\
\text { accommodating the flow }\end{array}$ \\
\hline Route discovery & & Broadcast RREQ messages \\
\hline Routing decisions & & Joint decisions based on MAC and Network layer \\
\hline Route nature & & On demand \\
\hline Link failure handling & Yes & Redirecting flow to another neighbor \\
\hline \multicolumn{3}{|l|}{ Protocol overhead } \\
\hline Route discovery packet size & Variable & Each intermediate node appends SOP list \\
\hline Best path selection & Yes & Based on the cumulative delay of the path \\
\hline Load balancing & Yes & A local coordination scheme is used \\
\hline Network topology & & Full mesh \\
\hline Control channel & Yes & $\begin{array}{l}\text { Exchanging spectrum opportunity among network } \\
\text { nodes }\end{array}$ \\
\hline Neighbor discovery & Yes & Channel scanning and beacon broadcast \\
\hline
\end{tabular}




\subsection{Multi-path Routing}

In multi-path routing multiple routes are discovered for any destination and then some best routes among discovered route are selected based on different parameters. Multi-path routing has many benefits such as fault tolerance, increased bandwidth and reduction of primary to secondary user interference. In the rest of the sub section we summarize a multi-path routing protocol and highlight its routing technique and its advantages.

\subsubsection{Multipath Routing and Spectrum Access (MRSA)}

Existing multi-path routing protocols for traditional wireless networks cannot be adapted in CRNs since they neither consider the diversity in spectrum availability nor coexistence of primary and secondary users. MRSA [16] is the first multi-path protocol for CRNs that minimizes the inter path contention and interference. It overcomes the interruption of primary users with minimum degradation by distributing the traffic of each flow over multiple paths.

For traffic distribution it uses round robin fashion that is not an effective technique. In MRSA "spectrum wise disjointness" concept is revised as if multiple paths do not have any interfering bands between them then these paths are spectrum wise disjointed. MRSA assumes that there will be total $\mathrm{N}$ channels for data traffic and signaling is delivered over these channels together with data traffic. It uses dynamic source routing (DSR) [21] mechanism for route discovery in which source node broadcasts an RREQ message with new RREQ_ID and attaches its band radio usage table (BRT). When an intermediate node receives RREQ before forwarding, it verifies if the RREQ_ID is new or if RREQ_ID is not new then it counts the hop count from source. If RREQ has fewer hop count than the previous RREQ it will append its BRT and then forwards it. Thus destination will receive the same RREQ from multiple paths. Thus it first assigns band and radio to each link then evaluates all the candidate paths by their available bandwidth. RERR message of DSR is extended to overcome the sudden arrival of primary user and it's the part of route recovery process.

\subsubsection{Advantages:}

The protocol constructs multiple paths to maximize spectrum wise disjointedness and to minimize contention and interference. It achieves higher throughput than other routing approaches and effectively utilizes the network resources. It also provides better resilience from the dynamic interruption of primary users.

Table 5: Summary of MRSA Routing Protocol.

\begin{tabular}{|l|l|l|}
\hline Objective and features & & Technique /explanation \\
\hline End-to end throughput & Yes & Using multi-radios and multipath \\
\hline Route discovery & & $\begin{array}{l}\text { Control channel, Broadcasting RREQ } \\
\text { message }\end{array}$ \\
\hline Routing decisions & & Does not base on cross layer \\
\hline Route nature & & Periodical \\
\hline Path failure handling & Yes & Used RERR messages \\
\hline Communication overhead & Yes & $\begin{array}{l}\text { Multiple flows on single radio therefore } \\
\text { band switching for each individual flow }\end{array}$ \\
\hline Route discovery packet size & Variable & Each node appends its ID and BRT \\
\hline Best path selection & Yes & Minimum hop count \\
\hline
\end{tabular}


International Journal of Wireless \& Mobile Networks (IJWMN) Vol. 3, No. 3, June 2011

\begin{tabular}{|l|l|l|}
\hline Data striping & Yes & Round robin fashion \\
\hline Network topology & & Mesh \\
\hline
\end{tabular}

\subsection{Tree Based Routing}

In tree based routing protocol a tree structured network is enabled by configuring a root. Tree based routing is centralized routing scheme which is controlled by a single network entity called base station. Thus network topology can be quickly constructed among CR station by configuring cognitive base station as root. In the rest of the sub section we summarize a tree based routing protocol and highlight its routing technique and its advantages.

\subsubsection{Cognitive Tree-based Routing (CTBR)}

Cognitive tree based routing (CTBR) [19] is an extension of tree based routing protocol (TBR) proposed for wireless mesh networks [17-18]. It uses global and local decision schemes for route calculation. Global decision scheme selects route with the best global end-to-end metric whereas local decision scheme selects the best interface with the least load. Multiple paths with the same global end-to-end metric can exist for the same destination. In this case the end-to-end path is selected based on the local decision scheme, which uses load measuring.

CTBR uses the routing procedure of TBR in which root periodically sends Root Announcement (RANN) message for tree formulation. Any node receives the RANN, caches the node whom it receives the RANN as its potential parent and then rebroadcast RANN with updated cumulative metric. The node will select a parent node from all potential parents based on the best metric (i.e. hope count) for the path to root. For registering with root every node that contains known path to root sends route reply (RREP). Any intermediate node that receives RREP forwards the message to its parent node as well updates its routing table by selecting source node of RREP as its destination. Thus at the end root constructs a tree as it has learnt all network nodes. To make TBR adaptable for CRNs a link quality metric has been introduced.

\subsubsection{Advantages:}

In this routing protocol they proposed a new cognitive aware link metric for computing global end-to-end metric.

Path is selected on the basics of global and local decision schemes. Average end-to-end delay of CTBR achieves about 5 times smaller than any hop count based scheme.

Table 6: Summary of CTBR Routing Protocol.

\begin{tabular}{|l|l|l|}
\hline Objective and features & \multicolumn{2}{|c|}{ Technique /explanation } \\
\hline End-to-end delay & & 5 times better than hop count scheme \\
\hline Route discovery & & $\begin{array}{l}\text { Broadcast Root Announcement (RANN) } \\
\text { message }\end{array}$ \\
\hline Routing decisions & & Does not based on cross layer \\
\hline Route nature & & Periodical \\
\hline link failure handling & No & \\
\hline Protocol overhead & Yes & Additional control bytes are transmitted \\
\hline Route discovery packet size & Fixed & $\begin{array}{l}\text { Every node updates a single field known as } \\
\text { "cumulative metric" }\end{array}$ \\
\hline
\end{tabular}


International Journal of Wireless \& Mobile Networks (IJWMN) Vol. 3, No. 3, June 2011

\begin{tabular}{|l|l|l|}
\hline Best path selection & Yes & $\begin{array}{l}\text { Path is selected on the basics of global and } \\
\text { local decision schemes }\end{array}$ \\
\hline Centralize & Yes & Single point of failure \\
\hline Network topology & & Tree \\
\hline
\end{tabular}

\section{Conclusion}

Routing in multi-hop CRNs is challenging task due to the diversity in channel availability and data rates, therefore currently researchers from all around the world are focusing to introduce some novel routing techniques for CRNs. In this paper, firstly we discussed the architecture and main routing differences and challenges for CRNs then we presented a comprehensive survey and analytical analysis of available routing techniques for CRNs. The routing techniques are classified into dynamic spectrum aware-based, multipath-based, local coordination-based, reactive source-based and tree-based depending on their protocol operation. We also highlighted the routing operation, as well as the advantages and disadvantages of each routing technique. Although many routing techniques look promising but mostly presented techniques uses the same routing metrics as conventional wireless networks. Therefore, there is need to design new metrics those exploits all the dynamic characteristics of CRNs and based on such metrics novel routing proposals should be presented.

\section{REFERENCES}

[1] B. Fette, Cognitive Radio Technology, Elsevier Inc. (2006).

[2] Yuan Yuan, Paramir Bahl, Ranveer Chandra, Thomas Moscibroda, Yunnan Wu. Allocating

Dynamic Time-Spectrum Blocks In Cognitive Radio Networks. In ACM MobiHoc 2007.

[3] Federal Communications Commission, Spectrum Policy Task Force Report, ET Docket No. 03222, Notice of Proposed Rule Making and Order2003.

[4] I. F. Akyildiz, W.-Y. Lee, M. C. Vuran, and S. Mohanty, NeXt generation/ dynamic spectrum access/cognitive radio wireless networks: A survey, Elsevier Computer Networks, 50(3), $2127-$ 2159 (Sept, 2006).

[5] R. W. Thomas, L. A. DaSilva, and A. B. MacKenzie, "Cognitive networks,” in Proc. of IEEE DySPAN2005, pp. 352-360, November 2005.

[6] Yang Xiao, Fei Hu “Cognitive radio networks”. 2009 Taylor \& Francis Group, LLC. ISBN: 978-1-4200-6420-9

[7] De Cout, D. S. J, et.al. A high-throughput path metric for multihop wireless routing. In Proc. of MobiCom (Sept. 2003).

[8] Ma, H. and Zheng, L. and Ma, X. and Luo," Spectrum-aware routing for multi-hop cognitive radio networks with a single transceiver", Proceedings of the Cognitive Radio Oriented Wireless Networks and Communications (CrownCom) 2008

[9] Kwang-Cheng, Chen, Ramjee Prasad, "Cognitive radio networks". 2009 John Wiley \& Sons Ltd. ISBN: 978-0-470-69689-7

[10] Sampath, A. and Yang, L. and Cao, L. and Zheng, H. and Zhao,” High Throughput Spectrumaware Routing for Cognitive Radio Networks. 
International Journal of Wireless \& Mobile Networks (IJWMN) Vol. 3, No. 3, June 2011

[11] Pefkianakis, I. and Wong, S.H.Y. and Lu,” SAMER: Spectrum Aware Mesh Routing in Cognitive Radio Networks", 3rd IEEE Symposium on New Frontiers in Dynamic Spectrum Access Networks, 2008. DySPAN 2008.

[12] Cheng, G. and Liu, W. and Li, Y. and Cheng," Spectrum aware on-demand routing in cognitive radio networks", 2nd IEEE International Symposium on New Frontiers in Dynamic Spectrum Access Networks, 2007. DySPAN 2007.pp.571-574.

[13] Ma, H. and Zheng, L. and Ma, X. and Luo,” Spectrum-aware routing for multi-hop cognitive radio networks with a single transceiver", Proceedings of the Cognitive Radio Oriented Wireless Networks and Communications (CrownCom) 2008.

[14] Khalife, H. and Ahuja, S. and Malouch, N. and Krunz," Routing in Opportunistic Cognitive Radio Networks".

[15] Yang, Z. and Cheng, G. and Liu, W. and Yuan, W. and Cheng.'Local coordination based routing and spectrum assignment in multi-hop cognitive radio networks", Mobile Networks and Applications 2008.

[16] Wang, X. and Kwon, T.T. and Choi,” A multipath routing and spectrum access (MRSA) framework for cognitive radio systems in multi-radio mesh networks" Proceedings of the 2009 ACM workshop on Cognitive radio networks 2009.

[17] IEEE 802.11s http://www.802wirelessworld.com/.

[18] A. Raniwala and T. C. Chiueh, "Architecture and algorithms for an IEEE802.11-based multichannel wireless mesh network,” in Proc. IEEE INFOCOM Conf., pp.2223-2234, 2005

[19] B. Zhang, Y. Takizawa, A. Hasagawa, A. Yamauchi, and S. Obana, "Tree-based routing protocol for cognitive wireless access networks, " in Proc. of IEEE Wireless Communications and Networking Conference2007.

[20] N1: C. E. Perkins and E. M. Royer, “Ad hoc on-demand distance vector routing," in Proc.of IEEE Workshop on Mobile Computing Systems and Applications, 1999.

[21] N2: D. B. Johnson, D.A. Maltz and Y. C. Hu, "The Dynamic Source Routing for mobile ad hoc networks,", draft-ietf-manet-dsr-09.txt, 2003. 